CARPATHIAN J. MATH.

Volume 38 (2022), No. 1,

Pages $201-215$
Online version at https : //www. carpathian. cunbm. utcluj.ro/

Print Edition: ISSN 1584 - 2851; Online Edition: ISSN 1843 - 4401

DOI: https://doi.org/10.37193/CJM.2022.01.16

Dedicated to the memory of Academician Mitrofan M. Choban (1942-2021)

\title{
Two parameter singular perturbation problems for sine-Gordon type equations
}

\author{
ANDREI PERJAN and GALina Rusu
}

ABSTRACT. In the real Sobolev space $H_{0}^{1}(\Omega)$ we consider the Cauchy-Dirichlet problem for sine-Gordon type equation with strongly elliptic operators and two small parameters. Using some $a$ priori estimates of solutions to the perturbed problem and a relationship between solutions in the linear case, we establish convergence estimates for the difference of solutions to the perturbed and corresponding unperturbed problems. We obtain that the solution to the perturbed problem has a singular behavior, relative to the parameters, in the neighbourhood of $t=0$.

\section{INTRODUCTION}

Let $\Omega \subset \mathbb{R}^{n}$ be an open bounded set with smooth boundary $\partial \Omega$. Consider the real Hilbert space $L^{2}(\Omega)$, endowed with the usual inner product $(u, v)_{L^{2}(\Omega)}=\int_{\Omega} u(x) v(x) d x$ and the norm $|\cdot|$, and the real Sobolev space $H_{0}^{1}(\Omega)$, endowed with the inner product $(u, v)_{H_{0}^{1}(\Omega)}=\int_{\Omega}(\nabla u(x), \nabla v(x))_{\mathbb{R}^{n}} d x$ and the norm $\|\cdot\|$.

We investigate the following boundary-value problem for sine-Gordon type equation

$$
\left\{\begin{array}{l}
\varepsilon \partial_{t}^{2} u_{\varepsilon \delta}(x, t)+\delta \partial_{t} u_{\varepsilon \delta}(x, t)+A u_{\varepsilon \delta}(x, t)+b \sin u_{\varepsilon \delta}(x, t)=f(x, t),(x, t) \in Q_{T}, \\
u_{\varepsilon \delta}(x, 0)=u_{0}(x), \partial_{t} u_{\varepsilon \delta}(x, 0)=u_{1}(x), \quad x \in \Omega \\
\left.u_{\varepsilon \delta}\right|_{\partial \Omega}=0, \quad t \geq 0
\end{array}\right.
$$

where $T>0, Q_{T}=\Omega \times(0, T), f \in L^{2}\left(Q_{T}\right), u_{0} \in V=H_{0}^{1}(\Omega), u_{1} \in H=L^{2}(\Omega), b \in \mathbb{R}$, $b \neq 0, \varepsilon, \delta$ are two small parameters and $A$ is a strongly elliptic operator of the type

$$
A: D(A)=H^{2}(\Omega) \cap H_{0}^{1}(\Omega) \mapsto L^{2}(\Omega), \quad A u=-\sum_{i, j=1}^{n} \partial_{x_{i}}\left(a_{i j}(x) \partial_{x_{j}} u(x)\right) .
$$

Namely, we suppose that the following conditions:

$$
(\text { HA })\left\{\begin{array}{l}
a_{i j} \in C^{1}(\bar{\Omega}), a_{i j}(x)=a_{j i}(x), \quad \forall x \in \bar{\Omega}, \\
\omega_{0}|\xi|^{2} \leq \sum_{i, j=1}^{n} a_{i j}(x) \xi_{i} \xi_{j} \leq \omega_{1}|\xi|^{2}, \quad \forall x \in \bar{\Omega}, \quad \forall \xi \in \mathbb{R}^{n}, 0<\omega_{0} \leq \omega_{1} .
\end{array}\right.
$$

are fulfilled.

Received: 18.04.2021. In revised form: 17.10.2021. Accepted: 24.10.2021

2010 Mathematics Subject Classification. 35B25, 35K15, 35L15, 34G10.

Key words and phrases. Sine-Gordon type equation, singular perturbation, boundary layer function, a priory estimate.

Corresponding author: Galina Rusu; rusugalinamoldova@gmail.com 
The interest for the sine-Gordon equation is explained by the various applications in differential geometry and engineering, including junctions between two superconductors, the motion of rigid pendular attached to a stretched wire, dislocations in crystals, nonlinear optics. For example, the solution of the equation

$$
u_{t t}(x, t)-u_{x x}(x, t)+\sin u(x, t)=0
$$

describes an angle of rotation of the pendulum in the case of mechanical transmission line.

Some numerical results for two-parameter singularly perturbed boundary problems for a linear case were established in [2], [5] and for the quasilinear case in [15]. Using the theory of differential inequalities, in [4] a two parameter boundary value problem for nonlinear equations of fourth order is considered. Some two parameter singular perturbations of linear boundary problems in abstract case have been carried out by W. M. Greenlee in [3].

The behavior of solutions to the abstract Cauchy-Dirichlet problem for sine-Gordon type equation with strongly elliptic operators and one parameter was established in [8]. In some previous works we obtained convergence estimates for abstract second order differential equations with one parameter and: linear operators [6], depending on time linear operators [10], Lipschitzian nonlinearities [8], monotone nonlinearities [9]; two small parameters and: linear operators [11], [12], depending on time linear operators [13], monotone nonlinearities [14].

Using similar specific techniques, the functional framework of the Sobolev space $H_{0}^{1}(\Omega)$ and the properties of the strongly elliptic operator, in this paper we investigate the behavior of solutions $u_{\varepsilon \delta}$ to the problem $\left(P_{\varepsilon \delta}\right)$ in two different cases:

(i) $\varepsilon \rightarrow 0$ and $\delta \geq \delta_{0}>0$, relative to the solutions to the following unperturbed system:

$$
\left\{\begin{array}{l}
\delta \partial_{t} l_{\delta}(x, t)+A l_{\delta}(x, t)+b \sin l_{\delta}(x, t)=f(x, t), \quad(x, t) \in Q_{T} \\
l_{\delta}(x, 0)=u_{0}(x), \quad x \in \Omega \\
\left.l_{\delta}\right|_{\partial \Omega}=0, \quad t \geq 0
\end{array}\right.
$$

(ii) $\varepsilon \rightarrow 0$ and $\delta \rightarrow 0$, relative to the solutions to the following unperturbed system:

$$
\left\{\begin{array}{l}
A v(x, t)+b \sin v(x, t)=f(x, t), \quad(x, t) \in Q_{T} \\
\left.v\right|_{\partial \Omega}=0, \quad t \geq 0
\end{array}\right.
$$

The problem $\left(P_{\varepsilon \delta}\right)$ is the abstract model of singularly perturbed problems of hyperbolicparabolic type in the case (i) and of the hyperbolic-parabolic-elliptic type in the case (ii).

The organization of this paper is as follows. At the beginning of the next section we present the theorems of existence and uniqueness of solutions to the problems $\left(P_{\varepsilon \delta}\right),\left(P_{\delta}\right)$ and some a priori estimates of their solutions. Then we present a relationship between solutions to the problem for the abstract linear second order differential equation and the corresponding solution to the problem for the first order equation. In the section 3 we present the main result of the paper. More precisely, we prove the convergence estimates of the difference of solutions to the problems $\left(P_{\varepsilon \delta}\right)$ and $\left(P_{\delta}\right)$ for $\varepsilon \rightarrow 0, \delta \geq \delta_{0}>0$ and also to the problems $\left(P_{\varepsilon \delta}\right)$ and $\left(P_{0}\right)$ for $\varepsilon \rightarrow 0, \delta \rightarrow 0$.

The framework of our investigations will be determined by conditions (HA) and also by the following condition

(HSG): $q_{0}=\omega_{0}-\lambda_{1}^{-1}|b|>0$, where $\lambda_{1}$ is the first eigenvalue of the spectral problem $-\Delta u=\lambda u,\left.u\right|_{\partial \Omega}=0$. 


\section{PRELIMINARIES}

In this section we remind some results about solvability of problems $\left(P_{\varepsilon \delta}\right),\left(P_{\delta}\right)$ and $\left(P_{0}\right)$. We prove some estimates of solutions to the problem $\left(P_{\varepsilon \delta}\right)$ which are uniform relative to the parameters. Finally, we present a relationship between the system $\left(P_{\varepsilon \delta}\right)$ and the corresponding system, governed by the first order equation, in the linear case when $\delta=1$. These estimates together with this relationship play a key role in establishing the behavior of solutions to the problem $\left(P_{\varepsilon \delta}\right)$ as $\varepsilon \rightarrow 0$ and $\delta \rightarrow 0$.

For this purpose we will define the abstract models associated with the problems $\left(P_{\varepsilon \delta}\right)$, $\left(P_{\delta}\right)$ and $\left(P_{0}\right)$. Since the solvability of these problems does not depend on the positive values of the parameters $\varepsilon$ and $\delta$, we will put $\varepsilon=\delta=1$. Thus, if the dependency on $x$ is suppressed and ' and " stand the time derivatives, we get the following abstract models:

for $\left(P_{\varepsilon \delta}\right)$

$$
\left\{\begin{array}{l}
u^{\prime \prime}(t)+u^{\prime}(t)+A u(t)+b \sin u(t)=f(t), \quad t \in(0, T), \\
u(0)=u_{0} \in V, \quad u^{\prime}(0)=u_{1} \in H
\end{array}\right.
$$

for $\left(P_{\delta}\right)$

$$
\left\{\begin{array}{l}
l^{\prime}(t)+A l(t)+b \sin l(t)=f(t), \quad t \in(0, T), \\
l(0)=u_{0} \in V
\end{array}\right.
$$

and for $\left(P_{0}\right)$

$$
A v+b \sin v=f(t), \quad t \in(0, T) .
$$

In (2.2), (2.3), (2.4), by $A$ is denoted the self-adjoint and positive definite extension on $V$ of the operator (1.1). This is possible, due the conditions (HA). The following theorems were inspired by the work [1] and are completely proved in [7].

Theorem 2.1. Let $T>0$. Assume that conditions (HA) are satisfied. If $u_{0} \in V, u_{1} \in H$ and $f \in L^{1}(0, T ; H)$, then there exists a unique function $u \in L^{2}(0, T ; V), u^{\prime} \in L^{2}(0, T ; H)$, $u^{\prime \prime} \in L^{2}\left(0, T ; V^{\prime}\right)$ such that $u$ satisfies the equation from (2.2) in the sence of distributions on $(0, T)$ and the initial conditions from (2.2). This function is called the strong solution to the problem (2.2).

If in adition, $u_{0} \in D(A), u_{1} \in V$ and $\left.f \in W^{1,1}(0, T ; H)\right)$, then $u \in W^{2, \infty}(0, T ; H)$, $u^{\prime} \in L^{\infty}(0, T ; V)$ and $A u \in L^{\infty}(0, T ; H)$.

Theorem 2.2. Let $T>0$. Assume that conditions (HA) are satisfied. If $u_{0} \in V$ and $f \in W^{1,1}(0, T ; H)$, then there exists a unique function $l \in W^{1,2}(0, T ; V)$ such that $l(t) \in D(A)$, a. e. $t \in(0, T), l$ satisfies the equation from (2.3) in the sense of distributions on $(0, T)$ and the initial conditions from (2.3). This function is called the strong solution to the problem (2.3). Moreover the function $t \mapsto(A l(t), l(t))$ is an absolutely continuous function on $[0, T]$ and

$$
\frac{d}{d t}(A l(t), l(t))=2\left(A l(t), l^{\prime}(t)\right), \quad \text { a. e. } \quad t \in[0, T] .
$$

Theorem 2.3. Let $T>0$ and $p>1$. Suppose that conditions (HA) and (HSG) are fulfilled. If $f \in W^{1, p}(0, T ; H)$, then the equation $A v+\sin (v)=f$ has a unique strong solution $v \in W^{1, p}(0, T ; H)$ and

$$
\|v\|_{W^{1, p}(0, T ; V)} \leq \frac{1}{q_{0}}\|f\|_{W^{1, p}(0, T ; H)}
$$


Proof. Denote by $\mathcal{A}=A+\sin (\cdot): D(A) \mapsto H$. Operator $\mathcal{A}$ is monotone. Indeed, in virtue of conditions (HA) and $q_{0}>0$, we have

$$
\begin{gathered}
\left(\mathcal{A} u_{1}-\mathcal{A} u_{2}, u_{1}-u_{2}\right)=\int_{\Omega} \sum_{i j=1}^{n} a_{i j}(x) \partial_{x_{i}}\left(u_{1}-u_{2}\right) \partial_{x_{j}}\left(u_{1}-u_{2}\right) d x+ \\
+b \int_{\Omega}\left(u_{1}-u_{2}\right)\left(\sin u_{1}-\sin u_{2}\right) d x \geq \\
\geq \omega_{0} \int_{\Omega}\left|\nabla\left(u_{1}-u_{2}\right)\right|^{2} d x-2|b| \int_{\Omega}\left|u_{1}-u_{2}\right|\left|\sin \left(\frac{u_{1}-u_{2}}{2}\right)\right| d x \geq
\end{gathered}
$$

$$
\geq \omega_{0} \int_{\Omega}\left|\nabla\left(u_{1}-u_{2}\right)\right|^{2} d x-|b| \int_{\Omega}\left|u_{1}-u_{2}\right|^{2} d x \geq q_{0}\left\|u_{1}-u_{2}\right\|^{2}, \quad \forall u_{1}, u_{2} \in D(A) .
$$

We will show that the operator $\mathcal{A}$ is even maximal monotone in $H$. For this purpose we consider the equation

$$
(\lambda I+\mathcal{A}) u=f,
$$

in $H$ with $f \in H$ and $\lambda>0$. Due to the condition (HA), there exists $(\lambda I+A)^{-1}: D\left((\lambda I+A)^{-1}\right)=H \mapsto R\left((\lambda I+A)^{-1}\right) \subseteq D(A)$ for any $\lambda>0$ and

$$
\left\|(\lambda I+A)^{-1}\right\|_{H \rightarrow H} \leq\left(\lambda+\omega_{0} \lambda_{1}\right)^{-1} .
$$

Then the equation (2.7) is equivalent to the equation

$$
u=(\lambda I+A)^{-1}(f-b \sin u) .
$$

In the real Hilbert space $V$ the equation (2.8) can be written in the form

$$
\mathcal{B}(u)=u,
$$

where $\mathcal{B}(u)=(\lambda I+A)^{-1}(f-b \sin u)$. The equality

$$
\left((A+\lambda I) A^{-1} u, u\right)=|u|^{2}+\lambda\left(A^{-1} u, u\right), \quad \forall u \in H,
$$

implies

$$
\left(A(A+\lambda I)^{-1} u, u\right)=\left|A(A+\lambda I)^{-1} u\right|^{2}+\lambda\left|A^{1 / 2}(A+\lambda I)^{-1} u\right|^{2}, \quad \forall u \in H,
$$

from which it follows that

$$
\left|A(A+\lambda I)^{-1} u\right| \leq|u|, \quad \forall u \in H .
$$

Consequently,

$$
\lambda \omega_{0}\left\|(A+\lambda I)^{-1} u\right\|^{2} \leq \lambda\left|A^{1 / 2}(A+\lambda I)^{-1} u\right|^{2} \leq|u|^{2}, \quad \forall u \in H
$$

and we have

$$
\left\|(\lambda I+A)^{-1}\right\|_{H \rightarrow V} \leq\left(\omega_{0} \lambda\right)^{-1 / 2} .
$$

Let us note that operator $\mathcal{B}$ is a contraction on $V$ for every $f \in H$ and every $\lambda>|b|^{2}\left(\lambda_{1} \omega_{0}\right)^{-1}$. Indeed,

$$
\begin{gathered}
|| \mathcal{B}\left(u_{1}\right)-\mathcal{B}\left(u_{2}\right)|| \leq|b|\left\|(\lambda I+A)^{-1}\left(\sin u_{1}-\sin u_{2}\right)\right\| \leq \\
\leq|b|\left(\omega_{0} \lambda\right)^{-1 / 2}\left|\sin u_{1}-\sin u_{2}\right| \leq|b|\left(\omega_{0} \lambda\right)^{-1 / 2}\left|u_{1}-u_{2}\right| \leq \\
\leq|b|\left(\omega_{0} \lambda \lambda_{1}\right)^{-1 / 2}|| u_{1}-u_{2}||, \quad \forall u_{1}, u_{2} \in V .
\end{gathered}
$$

According to Banach's Fixed Point Theorem, the equation (2.9) has a unique solution $u \in V$ for each $f \in H$. From (2.8) it follows that $u \in D(A)$. Thus, $R(\lambda I+\mathcal{A}) \supseteq H$ for $\lambda>|b|^{2}\left(\lambda_{1} \omega_{0}\right)^{-1}$. Therefore, according to Minty's Theorem, $\mathcal{A}$ is a maximal monotone 
operator if $|b|<\omega_{0} \lambda_{1}$. Since, by virtue of (2.6), the operator $\mathcal{A}$ is coercive, it is surjective i.e. $R(\mathcal{A})=H$. Hence there exists $\mathcal{A}^{-1}: H \mapsto D(A)$. Then the inequality (2.6) implies

$$
\| \mathcal{A}^{-1}\left(f_{1}\right)-\mathcal{A}^{-1}\left(f_{2}\right)|| \leq \frac{1}{q_{0}}\left|f_{1}-f_{2}\right|, \quad f_{1}, f_{2} \in H
$$

It means that the equation $\mathcal{A} v(t)=f(t)$ has a unique solution $v(t) \in D(A)$ for every $f(t) \in H$ and

$$
\|v(t)\| \leq\|v(0)\|+\frac{1}{q_{0}}\|f(t)-f(0)\| \leq\left\|\mathcal{A}^{-1}(f(0))\right\|+\frac{1}{q_{0}}|f(t)-f(0)|
$$

Thus, if $f \in W^{1, p}(0, T ; H)$, then $v \in L^{p}(0, T ; V)$. Finally, using (2.10) and Hölder's inequality, we get

$$
\begin{gathered}
\| v(t+h)-v(t)||^{p} \leq \frac{1}{q_{0}^{p}}|f(t+h)-f(t)|^{p}=\frac{1}{q_{0}^{p}}\left|\int_{t}^{t+h} f^{\prime}(\tau) d \tau\right|^{p} \leq \\
\leq \frac{h^{p-1}}{q_{0}^{p}} \int_{t}^{t+h}\left|f^{\prime}(\tau)\right|^{p} d \tau \quad t \in[0, T-h],
\end{gathered}
$$

or

$$
\begin{gathered}
\int_{0}^{T-h} \| v(t+h)-v(t)||^{p} d t \leq \frac{h^{p-1}}{q_{0}^{p}}\left[\int_{0}^{h} \tau\left|f^{\prime}(\tau)\right|^{p} d \tau+\int_{h}^{T-h} h\left|f^{\prime}(t)\right|^{p} d \tau\right]+ \\
+\frac{h^{p-1}}{q_{0}^{p}} \int_{T-h}^{T}(T-\tau)\left|f^{\prime}(\tau)\right|^{p} d \tau \leq \frac{h^{p}}{q_{0}^{p}} \int_{0}^{T}\left|f^{\prime}(\tau)\right|^{p} d \tau .
\end{gathered}
$$

In this case (see Theorem $1.18[1]) v \in W^{1, p}(0, T ; V)$ and

$$
\left\|v^{\prime}\right\|_{L^{p}(0, T ; V)} \leq \frac{1}{q_{0}}\left\|f^{\prime}\right\|_{L^{p}(0, T ; H)} .
$$

From (2.10) and (2.11) follows (2.5). Theorem 2.3 is proved.

Remark 2.1. $\lambda_{1}$ is the exact constant in the Poincare-Fridriechs's inequality. For example, if $\Omega=(a, b) \subset \mathbb{R}$, then $\lambda_{1}=\pi^{2} /(b-a)^{2}$.

For the further consideration we rewrite the problems $\left(P_{\varepsilon \delta}\right)$ and $\left(P_{\delta}\right)$ in the form:

$$
\left\{\begin{array}{l}
\mu U_{\mu}^{\prime \prime}(s)+U_{\mu}^{\prime}(s)+A U_{\mu}(s)+b \sin U_{\mu}(s)=F(s), \quad s \in(0, T / \delta), \\
U_{\mu}(0)=u_{0}, \quad U_{\mu}^{\prime}(0)=\delta u_{1},
\end{array}\right.
$$

and

$$
\left\{\begin{array}{l}
\mathcal{L}^{\prime}(s)+A \mathcal{L}(s)+b \sin \mathcal{L}(s)=F(s), \quad s \in(0, T / \delta), \\
\mathcal{L}(0)=u_{0},
\end{array}\right.
$$

where $U_{\mu}(s)=u_{\varepsilon \delta}(\delta s), \mathcal{L}(s)=l_{\delta}(s \delta), F(s)=f(s \delta)$ and $\mu=\varepsilon / \delta^{2}$.

In what follows we will prove some a priori estimates for solutions to the problems $\left(\mathcal{P}_{\mu}\right)$ and $\left(\mathcal{P}_{0}\right)$. To this end we need the following Lemma of Gronwall-Bellman type.

Lemma 2.1. Let $\psi \in L^{1}(a, b)(-\infty<a<b<\infty)$ with $\psi(s) \geq 0$ a. e. on $(a, b)$. If $h \in C([a, b])$ verifies

$$
h^{2}(t) \leq c^{2}+2 \int_{a}^{t} \psi(s) h(s) d s, \quad \forall t \in[a, b]
$$

then

also holds.

$$
|h(t)| \leq|c|+\int_{a}^{t} \psi(s) d s, \quad \forall t \in[a, b]
$$


Lemma 2.2. Suppose that conditions (HA) and (HSB) are fulfilled. If $u_{0} \in D(A), u_{1} \in V$, $F \in W^{1,1}(0, \infty ; H)$ then for any strong solution $U_{\mu}$ to the problem $\left(\mathcal{P}_{\mu}\right)$ the following estimate

$$
\begin{aligned}
& \mu\left\|U_{\mu}^{\prime \prime}\right\|_{L^{\infty}(0, s: H)}+\left\|U_{\mu}^{\prime}\right\|_{C([0, s]: H)}+\left\|U_{\mu}^{\prime}\right\|_{L^{2}(0, s: V)} \leq \\
& \leq C\left(q_{0}\right) \mathcal{M}_{0}(s), \quad s \geq 0, \quad \delta \in(0,1], \quad \mu \in\left(0, \mu_{0}\right],
\end{aligned}
$$

holds with $\mu_{0}=\left(\omega_{0} \lambda_{1}-|b|\right) /\left(2 b^{2}\right)$ and $\mathcal{M}_{0}(s)=\left|A u_{0}\right|+|| u_{1}||+|F(0)|+\left.|| F\right|_{W^{1,1}(0, s ; H)}$.

Proof. Let $U_{\mu h}(s)=U_{\mu}(s+h)-U_{\mu}(s)$ and denote by

$$
\begin{aligned}
E\left(U_{\mu h}, s\right)= & \left|U_{\mu h}(s)\right|^{2}+\left|U_{\mu h}(s)+2 \mu U_{\mu h}^{\prime}(s)\right|^{2}+4 \mu\left(A U_{\mu h}(s), U_{\mu h}(s)\right)+ \\
& +4 \mu \int_{0}^{s}\left|U_{\mu h}^{\prime}(\tau)\right|^{2} d \tau+4 \int_{0}^{s}\left(A U_{\mu h}(\tau), U_{\mu}(\tau)\right) d \tau .
\end{aligned}
$$

If $U_{\mu}$ is a strong solution to the problem $\left(\mathcal{P}_{\mu}\right)$, then

$$
\frac{d}{d s} E\left(U_{\mu h}, s\right)=4\left(F_{h}(s)-b\left(\sin \left(U_{\mu}(s)\right)\right)_{h}, U_{\mu h}(s)+2 \mu U_{\mu h}^{\prime}(s)\right), \quad s \geq 0 .
$$

Since

$$
\left.\mid b\left(\sin \left(U_{\mu}(s)\right)\right)_{h}, U_{\mu h}(s)+2 \mu U_{\mu h}^{\prime}(s)\right)\left.|\leq \mu| U_{\mu h}^{\prime}(s)\right|^{2}+|b| \lambda_{1}^{-1}(1+|b| \mu) \|\left. U_{\mu h}(s)\right|^{2},
$$

then integrating $(2.13)$ on $(0, s)$, we get

$$
\begin{gathered}
\left|U_{\mu h}(s)\right|^{2}+\left|U_{\mu h}(s)+2 \mu U_{\mu h}^{\prime}(s)\right|^{2}+2 q_{0} \int_{0}^{s}\left\|U_{\mu h}(\tau)\right\|^{2} d \tau \leq \\
\leq E\left(U_{\mu h}, 0\right)+4 \int_{0}^{s} \mid F_{h}\left(\tau|| U_{\mu h}(\tau)+2 \mu U_{\mu h}^{\prime}(\tau) \mid d \tau, \quad s \geq 0, \quad \text { for } \quad \mu \in\left(0, \mu_{0}\right] .\right.
\end{gathered}
$$

Applying Lemma 2.1 to the last inequality, we obtain

$$
\begin{aligned}
& \left|U_{\mu h}(s)\right|+\left|U_{\mu h}(s)+2 \mu U_{\mu h}^{\prime}(s)\right|+\left(\int_{0}^{s}|| U_{\mu h}(\tau) \|^{2} d \tau\right)^{1 / 2} \leq \\
& \leq C\left(q_{0}\right)\left[E^{1 / 2}\left(U_{\mu h}, 0\right)+\int_{0}^{s}\left|F_{h}(\tau)\right| d \tau\right], \quad s \geq 0, \quad \mu \in\left(0, \mu_{0}\right] .
\end{aligned}
$$

Under the conditions of this Lemma, due to the Theorem 2.1, we have that $U_{\mu}^{\prime} \in C([0, T] ; H),\left\|U_{\mu}\right\| \in C([0, T])$. Therefore, the following relations

$$
\left\{\begin{array}{l}
\left|h^{-1} U_{\mu h}(s)\right| \rightarrow\left|U_{\mu}^{\prime}(s)\right|, \quad h \rightarrow 0, \quad \text { in } \quad C([0, T]), \\
\left|h^{-1} U_{\mu h}(s)+2 \mu h^{-1} U_{\mu h}^{\prime}\right| \rightarrow\left|U_{\mu}^{\prime}(s)+2 \mu U_{\mu}^{\prime \prime}(s)\right|, \quad h \downarrow 0, \quad \text { a.e. } \quad s \in(0, T), \\
|| h^{-1} U_{\mu h}(s)|| \rightarrow|| U_{\mu}^{\prime}(s)||, \quad h \downarrow 0, \quad \text { a.e. } \quad s \in(0, T), \\
h^{-2} E\left(U_{\mu h}, 0\right) \rightarrow\left|\delta u_{1}\right|^{2}+\left|2\left(F(0)-A u_{0}-b \sin \left(u_{0}\right)\right)-\delta u_{1}\right|^{2}+4 \mu \delta^{2}\left\|u_{1}\right\|^{2}, h \downarrow 0
\end{array}\right.
$$

hold. Taking into account the relations (2.15), we divide (2.14) by $h$ and then pass to the limit in the obtained inequality, to get the estimate (2.12). Lemma 2.2 is proved. 
To establish the relationship between solutions to the problems $\left(\mathcal{P}_{\mu}\right)$ and $\left(\mathcal{P}_{0}\right)$ in the linear case we will define the kernel of transformation realizing this relationship.

For $\mu>0$ denote by

$$
K(t, \tau, \mu)=\frac{1}{2 \sqrt{\pi} \mu}\left(K_{1}(t, \tau, \mu)+3 K_{2}(t, \tau, \mu)-2 K_{3}(t, \tau, \mu)\right), \quad \forall \mu>0,
$$

where

$$
\begin{gathered}
K_{1}(t, \tau, \mu)=\exp \left\{\frac{3 t-2 \tau}{4 \mu}\right\} \lambda\left(\frac{2 t-\tau}{2 \sqrt{\mu t}}\right), \quad K_{2}(t, \tau, \mu)=\exp \left\{\frac{3 t+6 \tau}{4 \mu}\right\} \lambda\left(\frac{2 t+\tau}{2 \sqrt{\mu t}}\right), \\
K_{3}(t, \tau, \mu)=\exp \left\{\frac{\tau}{\mu}\right\} \lambda\left(\frac{t+\tau}{2 \sqrt{\mu t}}\right), \quad \lambda(s)=\int_{s}^{\infty} e^{-\eta^{2}} d \eta .
\end{gathered}
$$

In the following lemma we collected some properties of the kernel $K(t, \tau, \mu)$.

Lemma 2.3. [6] The function $K(t, \tau, \mu)$ is solution to the problem

$$
\left\{\begin{array}{l}
K_{t}(t, \tau, \mu)=\mu K_{\tau \tau}(t, \tau, \mu)-K_{\tau}(t, \tau, \mu), \quad \forall t>0, \quad \forall \tau>0, \\
\mu K_{\tau}(t, 0, \mu)-K(t, 0, \mu)=0, \quad \forall t \geq 0, \\
K(0, \tau, \mu)=\frac{1}{2 \mu} \exp \left\{-\frac{\tau}{2 \mu}\right\}, \quad \forall \tau \geq 0,
\end{array}\right.
$$

from $C([0, \infty) \times[0, \infty)) \cap C^{2}((0, \infty) \times(0, \infty))$ and possesses the following properties:

(i) $K(t, \tau, \mu)>0, \quad \forall t \geq 0, \quad \forall \tau \geq 0, \quad$ and $\quad \int_{0}^{\infty} K(t, \tau, \mu) d \tau=1, \quad \forall t \geq 0$;

(ii) Let $q \in[0,1]$. Then $\int_{0}^{\infty} K(t, \tau, \mu)|t-\tau|^{q} d \tau \leq C(\mu+\sqrt{\mu t})^{q}, \quad \forall \mu>0, \quad \forall t \geq 0$;

(iii) Let $p \in(1, \infty]$ and $f:[0, \infty) \rightarrow H, f(t) \in W^{1, p}(0, \infty ; H)$. Then

$$
\left|f(t)-\int_{0}^{\infty} K(t, \tau, \mu) f(\tau) d \tau\right| \leq C(p)\left\|f^{\prime}\right\|_{L^{p}(0, \infty ; H)}(\mu+\sqrt{\mu t})^{\frac{p-1}{p}}, \quad \forall \mu>0, \quad \forall t \geq 0 .
$$

Lemma 2.4. [6] Let $b=0$. Assume that $A: D(A) \subset H \rightarrow H$ is a linear, self-adjoint, positive definite operator and $F \in L^{\infty}(0, \infty ; H)$. If $U_{\mu}$ is a strong solution to the problem $\left(\mathcal{P}_{\mu}\right)$ with $U_{\mu} \in W^{2, \infty}(0, \infty ; H), A U_{\mu} \in L^{\infty}(0, \infty ; H)$, then the function $W_{\mu}$, defined by

$$
W_{\mu}(s)=\int_{0}^{\infty} K(s, \tau, \mu) U_{\mu}(\tau) d \tau
$$

is the strong solution to the problem

$$
\left\{\begin{array}{l}
W_{\mu}^{\prime}(s)+A W_{\mu}(s)=F_{0}(s, \mu), \quad \text { a.e. } \quad s>0, \quad \text { in } H, \\
W_{\mu}(0)=\varphi_{\mu}
\end{array}\right.
$$

where

$$
\begin{gathered}
F_{0}(s, \mu)=\frac{1}{\sqrt{\pi}}\left[2 \exp \left\{\frac{3 s}{4 \mu}\right\} \lambda\left(\sqrt{\frac{s}{\mu}}\right)-\lambda\left(\frac{1}{2} \sqrt{\frac{s}{\mu}}\right)\right] u_{1}+\int_{0}^{\infty} K(s, \tau, \mu) F(\tau) d \tau \\
\varphi_{\mu}=\int_{0}^{\infty} e^{-\tau} U_{\mu}(2 \mu \tau) d \tau
\end{gathered}
$$




\section{Behaviour of SOlutions TO THE PROBlem $\left(P_{\varepsilon \delta}\right)$}

In this section we will prove the main results concerning the behavior of the solutions to the problem $\left(P_{\varepsilon \delta}\right)$, in both cases: $\varepsilon \rightarrow 0$ and $\delta \geq \delta_{0}>0 ; \varepsilon \rightarrow 0$ and $\delta \rightarrow 0$, relative to solution to the corresponding unperturbed problem.

Theorem 3.4. Let $\Omega \subset \mathbb{R}^{n}$ be an open and bounded set with smooth boundary $\partial \Omega, T>0$ and $p \in(1, \infty]$. Assume that conditions (HA) and (HSG) are fulfilled. If $u_{0} \in H^{2}(\Omega) \cap H_{0}^{1}(\Omega)$, $u_{1} \in H_{0}^{1}(\Omega)$ and $f \in W^{1, p}\left(0, T ; L^{2}(\Omega)\right)$, then there exists a constant $C=C\left(T, p, \omega_{0}, \omega_{1}, \lambda_{1}, b\right)>0$ such that

$$
\begin{gathered}
\left\|u_{\varepsilon \delta}-l_{\delta}\right\|_{C\left([0, T] ; L^{2}(\Omega)\right)} \leq C \mathcal{M} \varepsilon^{\beta} \delta^{-5 / 2}, \quad \delta \in(0,1], \quad \varepsilon \in\left(0, \mu_{0} \delta^{2}\right], \\
\left\|u_{\varepsilon \delta}-l_{\delta}\right\|_{C\left([0, T] ; H_{0}^{1}(\Omega)\right)} \leq C \mathcal{M} \Theta(\varepsilon, \delta), \quad \delta \in(0,1], \quad \varepsilon \in\left(0, \mu_{0} \delta^{2}\right],
\end{gathered}
$$

where $u_{\varepsilon \delta}$ and $l_{\delta}$ are the strong solutions to the problems $\left(P_{\varepsilon \delta}\right)$ and $\left(P_{\delta}\right)$, respectively, $\mu_{0}$ is defined in (2.12), $\mathcal{M}=\left\|u_{0}\right\|_{H^{2}(\Omega)}+\left\|u_{1}\right\|_{H_{0}^{1}(\Omega)}+\|f\|_{W^{1, p}\left(0, T ; L^{2}(\Omega)\right)}$ and

$$
\beta=\left\{\begin{array}{l}
1 / 2, \text { if } f=0, \\
(p-1) /(2 p), \text { if } f \neq 0,
\end{array} \quad \Theta(\varepsilon, \delta)=\left\{\begin{array}{l}
\varepsilon^{1 / 4} \delta^{-3 / 2}, \text { if } f=0, \\
\varepsilon^{1 / 4} \delta^{-(3 p+2) /(2 p)}, \text { if } f \neq 0 \text { and } p \geq 2 \\
\varepsilon^{(p-1) /(2 p)} \delta^{-2}, \text { if } f \neq 0 \text { and } p \in(1,2)
\end{array}\right.\right.
$$

Proof. During this proof we will agree to denote by $C$ all constants $C\left(T, p, \omega_{0}, \omega_{1}, \lambda_{1}, b\right)$. We will also use the previously agreed notations: $D(A)=H^{2}(\Omega) \cap H_{0}^{1}(\Omega), V=H_{0}^{1}(\Omega)$ and $H=L^{2}(\Omega)$. For any $f \in W^{1, p}(0, T ; H)$ let us define the function $\widetilde{f}:[0, \infty) \mapsto H$ as follows:

$$
\tilde{f}(t)=\left\{\begin{array}{l}
f(t), \quad 0 \leq t \leq T \\
\frac{2 T-t}{T} f(T), \quad T<t \leq 2 T \\
0, \quad t>2 T
\end{array}\right.
$$

Then $\tilde{f}(t) \in W^{1, p}(0, T ; H)$ and, since $W^{1, p}(0, T ; H) \hookrightarrow C([0, T] ; H)$ continuously, we get

$$
\|\widetilde{f}\|_{W^{1, p}(0, \infty ; H)} \leq C(p) \max \left\{T, \frac{1}{T}\right\}\|f\|_{W^{1, p}(0, T ; H)} .
$$

If we denote by $\widetilde{U}_{\mu}$ the unique strong solution to the problem $\left(\mathcal{P}_{\mu}\right)$, defined on $(0, \infty)$ instead of $(0, S)$ with $S=T / \delta$ and $\widetilde{f}$ instead of $f$, then, from Theorem 2.1 and Lemma 2.2, it follows that $\tilde{U}_{\mu} \in W^{2, \infty}(0, \infty ; H) \cap W^{1,2}(0, \infty ; V), A \tilde{U}_{\mu} \in L^{\infty}(0, \infty ; H)$.

Moreover, the estimate (3.19) implies

$$
\|\widetilde{F}\|_{W^{1, p}(0, \infty ; H)} \leq C(p, T) \delta^{-1 / p}\|f\|_{W^{1, p}(0, T ; H)}, p \in(1, \infty], \forall \delta \in(0,1] .
$$

Due to the estimates (3.20) and Lemma 2.2, we obtain the following estimates

$$
\left\|\tilde{U}_{\mu}^{\prime}\right\|_{C([0, s] ; H)}+\left\|\tilde{U}_{\mu}^{\prime}\right\|_{L^{2}(0, s ; V)} \leq C \delta^{\gamma} \mathcal{M}, \quad s \geq 0, \quad \delta \in(0,1], \quad \mu \in\left(0, \mu_{0}\right]
$$

with $\mathcal{M}$ from(3.16), $\mu_{0}$ from (2.12) and

$$
\gamma= \begin{cases}0, & \text { if } \quad f=0 \\ -1 / p, & \text { if } \quad f \neq 0\end{cases}
$$

By Lemma 2.4, the function $W_{\mu}$, defined by

$$
W_{\mu}(s)=\int_{0}^{\infty} K(s, \tau, \mu) \widetilde{U}_{\mu}(\tau) d \tau,
$$


is a strong solution to the problem

$$
\left\{\begin{array}{l}
W_{\mu}^{\prime}(s)+A W_{\mu}(s)=\widetilde{F}_{0}(s, \mu), \quad \text { a.e. } \quad s>0, \quad \text { in } H, \\
W_{\mu}(0)=\varphi_{\mu},
\end{array}\right.
$$

where

$$
\widetilde{F}_{0}(s, \mu)=\delta f_{0}(s, \mu) u_{1}+\int_{0}^{\infty} K(s, \tau, \mu) \widetilde{F}(\tau) d \tau-\int_{0}^{\infty} K(s, \tau, \mu) B\left(\widetilde{U}_{\mu}(\tau)\right) d \tau,
$$

$$
f_{0}(s, \mu)=\frac{1}{\sqrt{\pi}}\left[2 \exp \left\{\frac{3 s}{4 \mu}\right\} \lambda\left(\sqrt{\frac{s}{\mu}}\right)-\lambda\left(\frac{1}{2} \sqrt{\frac{s}{\mu}}\right)\right], \quad \varphi_{\mu}=\int_{0}^{\infty} e^{-\tau} \widetilde{U}_{\mu}(2 \mu \tau) d \tau .
$$

Denote by $R(s, \mu)=\widetilde{\mathcal{L}}(s)-W_{\mu}(s)$, where $\widetilde{\mathcal{L}}$ is the strong solution to the problem $\left(\mathcal{P}_{0}\right)$ with $\tilde{F}$ instead of $F, T=\infty$ and $W_{\mu}$ is the strong solution to the problem (3.23). Then, due to Theorem 2.2, $R(\cdot, \mu) \in W_{\mathrm{loc}}^{1, \infty}(0, \infty ; H)$ and $R$ is a strong solution in $H$ to the problem

$$
\left\{\begin{array}{l}
R^{\prime}(s, \mu)+A R(s, \mu)+b \sin (\widetilde{\mathcal{L}}(s))-b \sin \left(W_{\mu}(s)\right)=\mathcal{F}(s, \mu), \text { a. e. } s>0, \\
R(0, \mu)=u_{0}-W_{\mu}(0),
\end{array}\right.
$$

where

$$
\mathcal{F}(s, \mu)=\tilde{F}(s)-\int_{0}^{\infty} K(s, \tau, \mu) \tilde{F}(\tau) d \tau-\delta f_{0}(s, \mu) u_{1}+
$$

$$
+b \sin \left(\widetilde{U}_{\mu}(s)\right)-b \sin \left(W_{\mu}(s)\right)+b \int_{0}^{\infty} K(s, \tau, \mu)\left[\sin \left(\widetilde{U}_{\mu}(\tau)\right)-\sin \left(\widetilde{U}_{\mu}(s)\right)\right] d \tau
$$

In what follows we need the following two Lemmas, which will be proved after the proof of the Theorem 3.4.

Lemma 3.5. Assume the conditions of Theorem 3.4 are fulfilled. Then for any $\delta \in(0,1]$ and any $\mu \in\left(0, \mu_{0}\right]$ the following estimates:

$$
\begin{aligned}
& \left\|\widetilde{U}_{\mu}-W_{\mu}\right\|_{C([0, s] ; H)} \leq C \mathcal{M} \mu^{1 / 2} \delta^{\gamma}(1+\sqrt{s}), \quad s \geq 0, \\
& \left\|\widetilde{U}_{\mu}-W_{\mu}\right\|_{C([0, s] ; V)} \leq C \mathcal{M} \mu^{1 / 4} \delta^{\gamma}\left(1+s^{1 / 4}\right), \quad s \geq 0 .
\end{aligned}
$$

are valid with $\mathcal{M}$ from (3.16) and $\gamma$ from (3.22).

Lemma 3.6. Assume that the conditions of Theorem 3.4 are fulfilled. Then for the strong solution to the problem (3.25) the following estimates

$$
\begin{gathered}
\|R\|_{C([0, s] ; H)} \leq C \mathcal{M} \mu^{\beta} \delta^{\gamma}\left(1+s^{3 / 2}\right), \quad s \geq 0, \quad \delta \in(0,1], \quad \mu \in\left(0, \mu_{0}\right], \\
\|R\|_{C([0, s] ; V)} \leq C \mathcal{M} \mu^{\beta} \delta^{\gamma}(1+s), \quad s \geq 0, \quad \delta \in(0,1], \quad \mu \in\left(0, \mu_{0}\right],
\end{gathered}
$$

are true with $\mathcal{M}$ from (3.16), $\beta$ from (3.18), $\gamma$ from (3.22) and $\mu_{0}$ from (2.12).

Finally, from these lemmas we deduce that

$$
\begin{aligned}
& \left\|\tilde{U}_{\mu}-\tilde{L}\right\|_{C([0, s] ; H)} \leq\left\|\tilde{U}_{\mu}-W_{\mu}\right\|_{C([0, s] ; H)}+\|R\|_{C([0, s] ; H)} \leq \\
& \leq C \mathcal{M} \mu^{\beta} \delta^{\gamma}\left(1+s^{3 / 2}\right), \quad s \geq 0, \quad \delta \in(0,1], \quad \mu \in\left(0, \mu_{0}\right],
\end{aligned}
$$

Since $U_{\mu}(s)=\tilde{U}_{\mu}(s), L(s)=\tilde{L}(s)$, for all $s \in[0, T / \delta], U_{\mu}(s)=u_{\varepsilon \delta}(\delta s)$ and $L(s)=l_{\delta}(\delta s)$, then we have

$$
\begin{gathered}
\left|u_{\varepsilon \delta}(t)-l_{\delta}(t)\right|=\left|u_{\varepsilon \delta}(\delta s)-l_{\delta}(\delta s)\right|=\left|\tilde{U}_{\mu}(s)-\tilde{L}(s)\right| \leq \\
\leq C \mathcal{M} \mu^{\beta} \delta^{\gamma-3 / 2}=C \mathcal{M} \varepsilon^{\beta} \delta^{-5 / 2}, \quad t \in[0, T], \quad \delta \in(0,1], \quad \varepsilon \in\left(0, \mu_{0} \delta^{2}\right] .
\end{gathered}
$$

Concequently, from (3.31) follows the estimate (3.16). 
In the same way, using (3.28) and (3.30) we get

$$
\begin{gathered}
\left\|u_{\varepsilon \delta}(t)-l_{\delta}(t)\right\|=\left\|u_{\varepsilon \delta}(\delta s)-l_{\delta}(\delta s)\right\|=\left\|\tilde{U}_{\mu}(s)-\tilde{L}(s)\right\| \leq \\
\leq\left\|\tilde{U}_{\mu}-W_{\mu}\right\|_{C([0, s] ; V)}+\|R\|_{C([0, s] ; V)} \leq \\
\leq C \mathcal{M} \mu^{\beta_{1}} \delta^{\gamma-1} \leq C \mathcal{M} \Theta(\varepsilon, \delta), \quad t \in[0, T], \quad \delta \in(0,1], \quad \varepsilon \in\left(0, \mu_{0} \delta^{2}\right],
\end{gathered}
$$

where

$$
\beta_{1}=\left\{\begin{array}{l}
1 / 4, \quad \text { if } \quad f=0, \\
\min \{1 / 4,(p-1) /(2 p)\}, \quad \text { if } \quad \text { and } \quad f \neq 0 .
\end{array}\right.
$$

Theorem 3.4 is proved.

Proof of Lemma 3.5. Proof of the estimate (3.27). Using properties (i) and (ii) from Lemma 2.3 and the estimate (3.21), we get

$$
\begin{gathered}
\left|\widetilde{U}_{\mu}(s)-W_{\mu}(s)\right| \leq \int_{0}^{\infty} K(s, \tau, \mu)\left|\widetilde{U}_{\mu}(s)-\widetilde{U}_{\mu}(\tau)\right| d \tau \leq \\
\leq \int_{0}^{\infty} K(s, \tau, \mu)\left|\int_{\tau}^{s}\right| \widetilde{U}_{\mu}^{\prime}(\xi)|d \xi| d \tau \leq C \mathcal{M} \delta^{\gamma} \int_{0}^{\infty} K(s, \tau, \mu)|\tau-s| d \tau \leq \\
\leq C \mathcal{M} \mu^{1 / 2} \delta^{\gamma}(1+\sqrt{s}), \quad s \geq 0, \quad \delta \in(0,1], \quad \mu \in\left(0, \mu_{0}\right] .
\end{gathered}
$$

Thus, the estimate (3.27) is proved.

Proof of the estimate (3.28). In the same way, using properties (i) and (ii) from Lemma 2.3 and the estimate (3.21), we get

$$
\begin{gathered}
\left\|\widetilde{U}_{\mu}(s)-W_{\mu}(\tau)\right\| \leq \int_{0}^{\infty} K(s, \tau, \mu)\left\|\widetilde{U}_{\mu}(s)-\widetilde{U}_{\mu}(\tau)\right\| d \tau \leq \\
\leq \int_{0}^{\infty} K(s, \tau, \mu)\left|\int_{\tau}^{s}\right|\left|\widetilde{U}_{\mu}^{\prime}(\xi)\right||d \xi| d \tau \leq \int_{0}^{\infty} K(s, \tau, \mu)\left|\int_{\tau}^{s}\left\|\widetilde{U}_{\mu}^{\prime}(\xi)\right\|^{2} d \xi\right|^{1 / 2}|\tau-s|^{1 / 2} d \tau \leq \\
\leq C \mathcal{M} \delta^{\gamma} \int_{0}^{\infty} K(s, \tau, \mu)|\tau-s|^{1 / 2} d \tau \leq C \mathcal{M} \mu^{1 / 4} \delta^{\gamma}\left(1+s^{1 / 4}\right), s \geq 0, \delta \in(0,1], \mu \in\left(0, \mu_{0}\right] .
\end{gathered}
$$

Thus, the estimate (3.28) is proved. Lemma 3.5 is proved.

Proof of Lemma 3.6. Proof of the estimate (3.29). Multiplying scalarly in $H$ the equation (3.25) by $R$ and then integrating on $(0, s)$ the obtained equality, we deduce

$$
|R(s, \mu)|^{2}+2 q_{0} \int_{0}^{s}(A R(\xi, \mu), R(\xi, \mu)) d \xi \leq|R(0, \mu)|^{2}+2 \int_{0}^{s}|\mathcal{F}(\xi, \mu)||R(\xi, \mu)| d \xi, \forall s \geq 0,
$$

where $\mathcal{F}(\xi, \mu)$ is defined by (3.26). Applying Lemma 2.1 to the last inequality, we get

$$
|R(s, \mu)| \leq|R(0, \mu)|+\int_{0}^{s}|\mathcal{F}(\xi, \mu)| d \xi, \quad \forall s \geq 0 .
$$

In what follows, we will estimate the right side of (3.32). Using (3.21), we get

$$
\begin{gathered}
|R(0, \mu)| \leq \int_{0}^{\infty} e^{-\tau}\left|\tilde{U}_{\mu}(2 \mu \tau)-u_{0}\right| d \tau \leq \int_{0}^{\infty} e^{-\tau} \int_{0}^{2 \mu \tau}\left|\tilde{U}_{\mu}^{\prime}(\xi)\right| d \xi d \tau \leq \\
\leq C \mathcal{M} \mu \delta^{\gamma} \int_{0}^{\infty} \tau e^{-\tau} d \tau=C \mathcal{M} \mu \delta^{\gamma}, \quad \delta \in(0,1], \quad \mu \in\left(0, \mu_{0}\right] .
\end{gathered}
$$

Let us estimate $|\mathcal{F}(t, \mu)|$. Using the property (iii) from Lemma 2.3 and (3.20), we have

$$
\left|\tilde{F}(s)-\int_{0}^{\infty} K(s, \tau, \mu) \tilde{F}(\tau) d \tau\right| \leq C\left\|\tilde{F}^{\prime}\right\|_{L^{p}(0, \infty ; H)}(\mu+\sqrt{\mu s})^{(p-1) / p} \leq
$$




$$
\leq C\left\|\tilde{f}^{\prime}\right\|_{L^{p}(0, T ; H)}(\delta(\mu+\sqrt{\mu s}))^{(p-1) / p}, \quad s \geq 0, \quad \delta \in(0,1], \quad \mu>0 .
$$

Since $e^{\xi} \lambda(\sqrt{\xi}) \leq C, \quad \forall \xi \geq 0$, then the following estimates

$$
\begin{gathered}
\int_{0}^{s} \exp \left\{\frac{3 \xi}{4 \mu}\right\} \lambda\left(\sqrt{\frac{\xi}{\mu}}\right) d \xi \leq C \mu \int_{0}^{\infty} e^{-\xi / 4} d \xi \leq C \mu, \quad s \geq 0, \quad \mu>0, \\
\int_{0}^{s} \lambda\left(\frac{1}{2} \sqrt{\frac{\xi}{\mu}}\right) d \xi \leq \mu \int_{0}^{\infty} \lambda\left(\frac{1}{2} \sqrt{\xi}\right) d \xi \leq C \mu, \quad s \geq 0, \quad \mu>0,
\end{gathered}
$$

hold. Consequently,

$$
\left|\delta \int_{0}^{s} f_{0}(\xi, \mu) u_{1} d \xi\right| \leq C \delta \mu\left|u_{1}\right|, \quad s \geq 0, \quad \mu>0, \quad \delta>0 .
$$

Using the estimates (3.27), we get the following estimates

$$
\begin{aligned}
& \quad\left|\sin \left(\widetilde{U}_{\mu}(s)\right)-\sin \left(W_{\mu}(s)\right)\right| \leq\left|\widetilde{U}_{\mu}(s)-W_{\mu}(s)\right| \leq \\
& \leq C \mathcal{M} \mu^{1 / 2} \delta^{\gamma}(1+\sqrt{s}) \quad s \geq 0, \quad \delta \in(0,1], \quad \mu \in\left(0, \mu_{0}\right], \\
& \quad \int_{0}^{\infty} K(s, \tau, \mu)\left|\sin \left(\widetilde{U}_{\mu}(\tau)\right)-\sin \left(\widetilde{U}_{\mu}(s)\right)\right| d \tau \leq \\
& \leq C \mathcal{M} \mu^{1 / 2} \delta^{\gamma}(1+\sqrt{s}) \quad s \geq 0, \quad \delta \in(0,1], \quad \mu \in\left(0, \mu_{0}\right] .
\end{aligned}
$$

Using (3.34), (3.35), (3.36) and (3.37), from (3.26) we obtain

$$
|\mathcal{F}(s, \mu)| \leq C \mathcal{M} \delta^{\gamma} \mu^{\beta}(1+\sqrt{s}), \quad s \geq 0, \delta \in(0,1], \quad \mu \in\left(0, \mu_{0}\right] .
$$

\section{Consequently,}

$$
\int_{0}^{s}|\mathcal{F}(\tau, \mu)| d \tau \leq C \mathcal{M} \delta^{\gamma} \mu^{\beta} s(1+\sqrt{s}), \quad s \geq 0, \delta \in(0,1], \quad \mu \in\left(0, \mu_{0}\right] .
$$

From (3.32), using (3.33) and (3.39) we get the estimate (3.29).

Proof of the estimate (3.30). From Theorem 2.2 it follows that $R \in W_{\mathrm{loc}}^{1,2}(0, \infty ; V)$, $R(s, \mu) \in D(A)$, a. e. $s>0$ and $A R \in L_{\mathrm{loc}}^{2}(0, \infty ; H)$.

Moreover the function $s \mapsto(A R(s, \mu), R(s, \mu))$ is an absolutely continuous function on $[0, S]$ for any $S>0$ and

$$
\frac{d}{d s}(A R(s, \mu), R(s, \mu))=2\left(A R(s, \mu), R^{\prime}(s, \mu)\right), \quad \text { a. e. } \quad s>0 .
$$

Note also that

$$
|A R(s, \mu)|^{2} \geq \omega_{0} \lambda_{1}(A R(s, \mu), R(s, \mu)), \quad \text { a. e. } \quad s>0,
$$

$$
(A R(s, \mu), \eta(s)) \leq(A R(s, \mu), R(s, \mu))^{1 / 2} \times(A \eta(t), \eta(t))^{1 / 2}, \quad \eta \in V, \quad \text { a. e. } \quad s>0 .
$$

Therefore

$$
\begin{gathered}
|A R(s, \mu)|^{2}-b\left(A R(s, \mu), \sin (\widetilde{\mathcal{L}}(s))-\sin \left(W_{\mu}(s)\right)\right) \geq \\
\geq|A R(s, \mu)|^{2}-|b||A R(s, \mu)||R(s, \mu)| \geq \frac{q_{0}}{\omega_{0}}|A R(s, \mu)|^{2} \quad \text { a. e. } \quad s>0 .
\end{gathered}
$$

We multiply the equation from (3.25) by $A R(s, \mu)$ and then integrate on $(0, s)$ to get (by using the above facts)

$$
(A R(s, \mu), R(s, \mu))+2 \frac{q_{0}}{\omega_{0}} \int_{0}^{s}|A R(\xi, \mu)|^{2} d \xi \leq
$$


or

$$
\leq(A R(0, \mu), R(0, \mu))+2 \int_{0}^{s}|\mathcal{F}(\xi, \mu)||A R(\xi, \mu)| d \xi, \quad \forall s \geq 0
$$

$$
\begin{gathered}
\|R(s, \mu)\|^{2} \leq \omega_{0}^{-1}(A R(s, \mu), R(s, \mu)) \leq \\
\leq \omega_{0}^{-1}(A R(0, \mu), R(0, \mu))+\left(2 q_{0}\right)^{-1} \int_{0}^{s}|\mathcal{F}(\xi, \mu)|^{2} d \xi, \quad \forall s \geq 0,
\end{gathered}
$$

The last inequality implies that

$$
\|R(s, \mu)\| \leq C\left[\|R(0, \mu)\|+\|\mathcal{F}\|_{L^{2}(0, s: H)}\right], \quad \forall s \geq 0 .
$$

In what follows we will estimate all the terms from the right side of inequalities (3.43).

Similarly as the estimate (3.33) was obtained, we deduce that

$$
\|R(0, \mu)\| \leq \int_{0}^{\infty} e^{-\tau} \int_{0}^{2 \mu \tau}\left\|\tilde{U}_{\mu}^{\prime}(\xi)\right\| d \xi d \tau \leq \int_{0}^{\infty} e^{-\tau}\left\|\tilde{U}_{\mu}^{\prime}\right\|_{L^{2}(0,2 \mu \tau ; H)}(2 \mu \tau)^{1 / 2} d \tau \leq
$$

$$
\leq C \mathcal{M} \mu^{1 / 2} \delta^{\gamma} \int_{0}^{\infty} \tau e^{-\tau} d \tau=C \mathcal{M} \mu^{1 / 2} \delta^{\gamma}, \quad \delta \in(0,1], \quad \mu \in\left(0, \mu_{0}\right] .
$$

Using (3.38), we have

$$
\|\mathcal{F}(\cdot, \mu)\|_{L^{2}(0, s ; H)} \leq C \mathcal{M} \delta^{\gamma} \mu^{\beta} s^{1 / 2}\left(1+s^{1 / 2}\right), \quad s \geq 0, \delta \in(0,1], \quad \mu \in\left(0, \mu_{0}\right] .
$$

Finally, using (3.44) and (3.45), from (3.43) we get (3.30). Thus, Lemma 3.6 is proved. In what follows we will investigate the behavior of solutions to the problem $\left(P_{\delta}\right)$ as $\delta \rightarrow 0$.

Theorem 3.5. Let $\Omega \subset \mathbb{R}^{n}$ be an open and bounded set with boundary $\partial \Omega \in C^{1}, T>0$ and $p \in(1, \infty]$. Assume that conditions (HA) and (HSG) are fulfilled. If $u_{0} \in H^{2}(\Omega) \bigcap H_{0}^{1}(\Omega)$ and $f \in W^{1,1}\left(0, T ; L^{2}(\Omega)\right)$, then there exists a constant $C=C\left(T, p, \omega_{0}, \omega_{1}, \lambda_{1}, b\right)>0$ such that

$$
\begin{aligned}
& \left\|l_{\delta}(t)-v(t)\right\|_{L^{2}(\Omega)} \leq\left\|h_{0}\right\|_{L^{2}(\Omega)} e^{-\lambda_{1} q_{0} t / \delta}+C \mathcal{M} \delta^{(p-1) / p}, \quad t \in(0, T], \quad \delta \in(0,1), \\
& \left\|l_{\delta}(t)-v(t)\right\|_{H_{0}^{1}(\Omega)} \leq\left\|h_{0}\right\|_{H_{0}^{1}(\Omega)} e^{-\lambda_{1} q_{0} t / \delta}+C \mathcal{M} \delta^{(p-1) / p}, \quad t \in(0, T], \quad \delta \in(0,1),
\end{aligned}
$$

where $l_{\delta}$ and $v$ are the strong solutions to the problems $\left(P_{\delta}\right)$ and $\left(P_{0}\right)$, respectively, and $h_{0}=u_{0}-(A+\sin (\cdot))^{-1} f(0)$.

Proof. Proof of the estimate (3.46). Denote by $R_{1}(t, \delta)=l_{\delta}(t)-v(t)$, where $l_{\delta}$ is the strong solution to the problem $\left(P_{\delta}\right)$ and $v$ is the strong solution to the problem $\left(P_{0}\right)$. Then $R_{1}(t, \delta)$ is the strong solution to the problem

$$
\left\{\begin{array}{l}
\delta R_{1}^{\prime}(t, \delta)+A R_{1}(t, \delta)=-\delta v^{\prime}(t)-b \sin \left(l_{\delta}(t)\right)+b \sin (v(t)), \quad t \in(0, T), \\
R_{1}(0, \delta)=u_{0}-(A+\sin (\cdot))^{-1} f(0) .
\end{array}\right.
$$

Multiplying equation from (3.48) scalarly in $H$ by $R_{1}$, we obtain the equality

$$
\begin{gathered}
\delta \frac{d}{d t}\left|R_{1}(t, \delta)\right|^{2}+2\left(A R_{1}(t, \delta), R_{1}(t, \delta)\right)= \\
=-2 \delta\left(v^{\prime}(t), R_{1}(t, \delta)\right)+2 b\left(\sin (v(t))-\sin \left(l_{\delta}(t)\right), R_{1}(t, \delta)\right), \quad t \in(0, T) .
\end{gathered}
$$

Then, using conditions (HA) and (HSG), we get

$$
\delta \frac{d}{d t}\left|R_{1}(t, \delta)\right|^{2}+2\left(\omega_{0} \lambda_{1}-|b|\right)\left|R_{1}(t, \delta)\right|^{2} \leq 2 \delta\left|v^{\prime}(t)\right|\left|R_{1}(t, \delta)\right|, \quad t \in(0, T) .
$$


From the last inequality we get

$$
\frac{d}{d t}\left|R_{1}(t, \delta) e^{\lambda_{1} q_{0} t / \delta}\right|^{2} \leq 2\left|v^{\prime}(t) e^{\lambda_{1} q_{0} t / \delta}\right|\left|R_{1}(t, \delta) e^{\lambda_{1} q_{0} t / \delta}\right|, \quad t \in(0, T) .
$$

Integrating this inequality on $(0, t)$, we obtain

$$
\left|R_{1}(t, \delta) e^{\lambda_{1} q_{0} t / \delta}\right|^{2} \leq\left|R_{1}(0, \delta)\right|^{2}+2 \int_{0}^{t}\left|v^{\prime}(\tau) e^{\lambda_{1} q_{0} \tau / \delta}\right|\left|R_{1}(\tau, \delta) e^{\lambda_{1} q_{0} \tau / \delta}\right| d \tau, \quad t \in[0, T] .
$$

Applying Lemma 2.1 to the last inequality and using (2.5), we get the estimate

$$
\begin{gathered}
\left|R_{1}(t, \delta)\right| \leq\left|R_{1}(0, \delta)\right| e^{-\lambda_{1} q_{0} t / \delta}+\int_{0}^{t} e^{-\lambda_{1} q_{0}(t-\tau) / \delta}\left|v^{\prime}(\tau)\right| d \tau \leq \\
\leq\left|R_{1}(0, \delta)\right| e^{-\lambda_{1} q_{0} t / \delta}+\left(\frac{\delta}{\lambda_{1} q_{0}}\right)^{(p-1) / p}\left\|v^{\prime}\right\|_{L^{p}(0, T ; H)} \leq \\
\leq\left|R_{1}(0, \delta)\right| e^{-\lambda_{1} q_{0} t / \delta}+C\left(\frac{\delta}{\lambda_{1} q_{0}}\right)^{(p-1) / p}\|f\|_{W^{1, p}(0, T ; H)}, \quad t \in[0, T] .
\end{gathered}
$$

from which follows (3.46).

Proof of the estimate (3.47). As in the proof of the estimate (3.30), from Theorem 2.2 it follows that $R_{1} \in W_{\mathrm{loc}}^{1,2}(0, \infty ; V), R_{1}(t, \delta) \in D(A)$, a. e. $t>0, A R_{1} \in L_{\mathrm{loc}}^{2}(0, \infty ; H)$ and the function $t \mapsto\left(A R_{1}(t, \delta), R_{1}(t, \delta)\right)$ is an absolutely continuous function on $[0, T]$ for any $T>0$. Moreover the relationships (3.40), (3.41), (3.42) are true, in which $R(s, \mu)$ and $\eta(t)$ are replaced by $R_{1}(t, \delta)$ and by $v^{\prime}(t)$, respectively. Therefore

$$
\begin{gathered}
\left|A R_{1}(t, \delta)\right|^{2}-b\left(A R_{1}(t, \delta), \sin \left(l_{\delta}(t)\right)-\sin (v(t))\right) \geq \\
\geq \frac{q_{0}}{\omega_{0}}\left|A R_{1}(t, \delta)\right|^{2} \geq q_{0} \lambda_{1}\left(A R_{1}(t, \delta), R_{1}(t, \delta)\right), \quad \text { a. e. } \quad t>0,
\end{gathered}
$$

and, due to the estimate (2.5),

$$
\begin{gathered}
\left|\left(v^{\prime}(t), A R_{1}(t, \delta)\right)\right| \leq\left(A R_{1}(t, \delta), R_{1}(t, \delta)\right)^{1 / 2}\left(A v^{\prime}(t), v^{\prime}(t)\right)^{1 / 2} \leq \\
\leq \omega_{1}^{1 / 2}\left(A R_{1}(t, \delta), R_{1}(t, \delta)\right)^{1 / 2}\left\|v^{\prime}(t)\right\|, \quad \text { a. e. } \quad t>0 .
\end{gathered}
$$

Denote $y(t)=\left(A R_{1}(t, \delta), R_{1}(t, \delta)\right)^{1 / 2}$. Multiplying in $H$ the equation from (3.48) by $A R_{1}$ and using (3.49) and (3.50), we get the inequality

$$
\frac{d}{d t}\left|e^{q_{0} \lambda_{1} t / \delta} y(t)\right|^{2} \leq 2 \frac{\omega_{1}^{1 / 2}}{q_{0}} e^{2 q_{0} \lambda_{1} t / \delta} y(t)\left\|v^{\prime}(t)\right\|, \quad \text { a. e. } \quad t>0
$$

from which after integration, we obtain

$$
\left|e^{q_{0} \lambda_{1} t / \delta} y(t)\right|^{2} \leq|y(0)|^{2}+2 \frac{\omega_{1}^{1 / 2}}{q_{0}} \int_{0}^{t} e^{q_{0} \lambda_{1} s / \delta} y(s) e^{q_{0} \lambda_{1} s / \delta}\left\|v^{\prime}(s)\right\| d s, \quad t>0 .
$$

Applying Lemma 2.1 and the estimate (2.5), from the last inequality we get

$$
\begin{gathered}
y(t) \leq e^{-q_{0} \lambda_{1} t / \delta} y(0)+C \int_{0}^{t} e^{-q_{0} \lambda_{1}(t-s) / \delta}\left\|v^{\prime}(s)\right\| d s \leq \\
\leq y(0) e^{-q_{0} \lambda_{1} t / \delta}+\left(\frac{\delta}{\lambda_{1} q_{0}}\right)^{(p-1) / p}\left\|v^{\prime}\right\|_{L^{p}(0, T ; H)} \leq \\
\leq y(0) e^{-q_{0} \lambda_{1} t / \delta}+C\left(\frac{\delta}{\lambda_{1} q_{0}}\right)^{(p-1) / p}\|f\|_{W^{1, p}(0, T ; H)}, \quad t \in[0, T] .
\end{gathered}
$$


As

$$
\left\|R_{1}(t, \delta)\right\| \leq\left(\omega_{0}\right)^{-1 / 2} y(t) \quad \text { and } \quad y(0) \leq\left(\omega_{1}\right)^{1 / 2}\left\|R_{1}(0, \delta)\right\|,
$$

then the inequality (3.51) implies (3.47).

Theorem 3.5 is proved.

From Theorems 3.4 and 3.5 immediately follows the following theorem.

Theorem 3.6. Let $\Omega \subset \mathbb{R}^{n}$ be an open and bounded set with boundary $\partial \Omega \in C^{1}, T>0$ and $p \in(1, \infty]$. Assume that conditions (HA) and (HSG) are fulfilled. If $u_{0} \in H^{2}(\Omega) \bigcap H_{0}^{1}(\Omega)$, $u_{1} \in H_{0}^{1}(\Omega)$ and $f \in W^{1, p}\left(0, T ; L^{2}(\Omega)\right)$, then there exists a constant $C=C\left(T, p, \omega_{0}, \omega_{1}, \lambda_{1}, b\right)>0$ such that

$$
\begin{gathered}
\left\|u_{\varepsilon \delta}-v\right\|_{C\left([0, T] ; L^{2}(\Omega)\right)} \leq\left\|h_{0}\right\|_{L^{2}(\Omega)} e^{-\lambda_{1} q_{0} t / \delta}+C \mathcal{M}\left[\varepsilon^{\beta} \delta^{-5 / 2}+\delta^{(p-1) / p}\right], \\
\left\|u_{\varepsilon \delta}-v\right\|_{C\left([0, T] ; H_{0}^{1}(\Omega)\right)} \leq\left\|h_{0}\right\|_{H_{0}^{1}(\Omega)} e^{-\lambda_{1} q_{0} t / \delta}+C \mathcal{M}\left[\Theta(\varepsilon, \delta)+\delta^{(p-1) / p}\right],
\end{gathered}
$$

$\delta \in(0,1], \varepsilon \in\left(0, \mu_{0} \delta^{2}\right]$, where $u_{\varepsilon \delta}$ and $v$ are the strong solutions to the problems $\left(P_{\varepsilon \delta}\right)$ and $\left(P_{0}\right)$, respectively, $\mathcal{M}$ is from (3.16), $\Theta$ and $\beta$ are from (3.18), $h_{0}$ is from (3.47) and $\mu_{0}$ is from (2.12).

\section{CONCLUSIONS}

1. Under the conditions of Theorem 3.4 for $\delta \geq \delta_{0}>0$, it follows that

$$
\left\|u_{\varepsilon \delta}-l_{\delta}\right\|_{C\left([0, T] ; L^{2}(\Omega)\right)} \leq C \mathcal{M} \varepsilon^{\beta},
$$

and

$$
\left\|u_{\varepsilon \delta}-l_{\delta}\right\|_{C\left([0, T] ; H_{0}^{1}(\Omega)\right)} \leq C \mathcal{M} \Theta(\varepsilon, 1),
$$

with $C=C\left(T, p, \omega_{0}, \lambda_{1}, b, \delta_{0}\right)>0, \mathcal{M}$ from (3.16), $\beta$ and $\Theta(\varepsilon, 1)$ from (3.18).

Consequently, for $\delta \geq \delta_{0}>0$, the solutions $u_{\varepsilon \delta}$ to the problem $\left(P_{\varepsilon \delta}\right)$ have a regular behavior, as $\varepsilon \rightarrow 0$, relative to the solution $l_{\delta}$ to the problem $\left(P_{\delta}\right)$ in the neighborhood of $t=0$ in the considered spaces.

2. Under the conditions of Theorem 3.5 it follows that for every $t_{0}>0$ and $T>t_{0}$,

$$
l_{\delta} \rightarrow v \quad \text { in } C\left(\left[t_{0}, T\right] ; H_{0}^{1}(\Omega)\right) \quad \text { as } \delta \rightarrow 0 .
$$

If the concordance condition $f(0)=A u_{0}+b \sin u_{0}$ is satisfied, then

$$
l_{\delta} \rightarrow v \quad \text { in } \quad C\left([0, T] ; H_{0}^{1}(\Omega)\right) \quad \text { as } \quad \delta \rightarrow 0 .
$$

If the concordance condition $f(0)=A u_{0}+b \sin u_{0}$ is not satisfied, the solutions $l_{\delta}$ to the problem $\left(P_{\delta}\right)$ have a singular behavior, as $\delta \rightarrow 0$, relative to the solution $v$ to the problem $\left(P_{0}\right)$ in the neighborhood of $t=0$.

3. Under the conditions of Theorem 3.6 it follows that for every $t_{0}>0$ and $T>t_{0}$,

$$
u_{\varepsilon \delta} \rightarrow v \text { in } C\left(\left[t_{0}, T\right] ; L^{2}(\Omega)\right) \text { as } \varepsilon^{\beta} \delta^{-5 / 2}+\delta^{(p-1) / p} \rightarrow 0,
$$

and

$$
u_{\varepsilon \delta} \rightarrow v \quad \text { in } \quad C\left(\left[t_{0}, T\right] ; H_{0}^{1}(\Omega)\right) \quad \text { as } \quad \Theta(\varepsilon, \delta)+\delta^{(p-1) / p} \rightarrow 0 .
$$

If the concordance condition $f(0)=A u_{0}+b \sin u_{0}$ is satisfied, then

$$
u_{\varepsilon \delta} \rightarrow v \quad \text { in } C\left([0, T] ; L^{2}(\Omega)\right) \text { as } \quad \varepsilon^{\beta} \delta^{-5 / 2}+\delta^{(p-1) / p} \rightarrow 0,
$$

and

$$
u_{\varepsilon \delta} \rightarrow v \quad \text { in } \quad C\left([0, T] ; H_{0}^{1}(\Omega)\right) \quad \text { as } \quad \Theta(\varepsilon, \delta)+\delta^{(p-1) / p} \rightarrow 0 .
$$

If the concordance condition $f(0)=A u_{0}+b \sin u_{0}$ is not satisfied, the solutions $u_{\varepsilon \delta}$ to the problem $\left(P_{\varepsilon \delta}\right)$ have a singular behavior, as $\varepsilon \rightarrow 0, \delta \rightarrow 0$, relative to the solution $v$ to the problem $\left(P_{0}\right)$ in the neighborhood of $t=0$. 
Acknowledgements. The second author Researches supported by the Project 20.80009.5007.25

\section{REFERENCES}

[1] Barbu, V. Nonlinear Differential Equations of Monotone Types in Banach Spaces. Springer-Verlag, New York, 2010.

[2] Brdar, M.; Zarin, H. A singularly perturbed problem with two parameters on a Bakhvalov-type mesh. J. Comput. Appl. Math. 292 (2016), no. 3, 307-319.

[3] Greenlee, W. On Two Parameter Singular Perturbation Of Linear Boundary Value Problems. Proceedings of the American Mathematical Society 27 (1971), no. 2, 268-274.

[4] Jiaqi, M. Singularly Perturbed Solution of Boundary Value Problem for Nonlinear Equations of Fourth Order With Two Parameters. Advances in Mathematics 39 (2010), no. 6, 736-740.

[5] Linss, T. A posteriori error estimation for a singularly perturbed problem with two small parameters. Int. J. Numer. Anal. Model. 39 (2010), no. 6, 491-506.

[6] Perjan, A. Linear singular perturbations of hyperbolic-parabolic type. Bul. Acad. Stiinte Repub. Mold. Mat. 42 (2003), no. 2, 95-112.

[7] Perjan, A. Singularly perturbed boundary value problems for evolution differential equations D. Sc. Thesis, Moldova State University, 2008. (In Romanian)

[8] Perjan, A.; Rusu, G. Convergence estimates for abstract second-order singularly perturbed Cauchy problems with Lipschitzian nonlinearities. Asymptot. Anal. 74 (2011), no. 3-4, 135-165.

[9] Perjan, A.; Rusu, G. Convergence estimates for abstract second order singularly perturbed Cauchy problems with monotone nonlinearities. Ann. Acad. Rom. Sci. Ser. Math. Appl. 4 (2012), no. 2, 128-182.

[10] Perjan, A.; Rusu, G. Limits of solutions to the singularly perturbed abstract hyperbolic-parabolic system. Bul. Acad. Stiinte Repub. Mold. Mat. 49 (2014), no. 3, 49-64.

[11] Perjan, A.; Rusu, G. Convergence estimates for abstract second-order singularly perturbed Cauchy problems with Lipschitz nonlinearities. Asymptot. Anal. 97 (2016), no. 3-4, 337-349.

[12] Perjan, A.; Rusu, G. Singularly perturbed problems for abstract differential equations of second order in Hilbert spaces. in: New trends in differential equations, control theory and optimization Word Scientific, New Jersey (2016), 277-293.

[13] Perjan, A.; Rusu, G., Abstract linear second order differential equations with two small parameters and depending on time operators. Carpathian J. Math. 33 (2017), no. 2, 233 -246.

[14] Perjan, A.; Rusu, G. Convergence estimates for abstract second order differential equations with two small parameters and monotone nonlinearities. Topol. Methods Nonlinear Anal. 54 (2019), no. 2B, 1093-1110.

[15] Vulanovic, R. A Higher-Order Scheme for Quasilinear Boundary Value Problems with Two Small Parameters. Computing 67 (2001), 287-303.

DEPARTMENT OF MATHEMATICS

MOLDOVA STATE UNIVERSITY

A. Mateevici 60, MD 2009, Chişinău, Republic of Moldova

Email address: aperjan1948@gmail.com

Email address: rusugalinamoldova@gmail.com 